\title{
Preface: Special Issue on Uncertain Reasoning
}

\author{
Yang Xiang • Kevin Grant
}

Received: 6 November 2009 / Accepted: 23 November 2009 / Published online: 4 December 2009

(C) Springer Science + Business Media B.V. 2009

This special issue contains selected and extended papers from the Uncertain Reasoning Special Track at the 2008 International Florida Artificial Intelligence Research Society Conference (FLAIRS). The Uncertain Reasoning Special Track is the oldest track in FLAIRS conferences, running annually since 1996. Over the years, the track has been established as a key event where researchers on broad issues related to reasoning under uncertainty, following a variety of alternative paradigms, formalisms and methodologies, come together to exchange insights and to influence each other's work.

The 2008 track also features a special session dedicated to Henry E. Kyburg Jr., a renowned, respected professor of computer science and philosophy, and a founding member and long time contributor of the track, who passed away October 30, 2007. During a 43 year academic career, he made significant contributions to the field of uncertain reasoning. He published numerous articles and books on topics such as inductive logic, statistical reasoning, probability, and epistemology. Henry loved to see a thousand flowers bloom. He was interested in belief measures, uncertain inference, nonmonotonicity and combining logic with probability. Papers included in this special issue are assembled in this very spirit.

In this special issue, Palacios-Alonso, Brizuela, and Sucar study dynamic naive Bayesian classifiers. They propose an evolutionary optimization algorithm for designing such classifiers. The design methodology is applied to hand gesture recognition. Experimental results show that the evolved network has higher average classification accuracy than the basic dynamic naive Bayesian classifier and a hidden Markov model.

\footnotetext{
Y. Xiang $(\varangle)$

University of Guelph, Guelph, Canada

e-mail: yxiang@cis.uoguelph.ca

K. Grant

University of Lethbridge, Lethbridge, Canada

e-mail: kevin@cs.uleth.ca
} 
One of the main objective of nonmonotonic reasoning is to cope with the presence of exceptions in knowledge based systems. The paper by Benferhat, Yahi and Drias focuses on the minimum specificity principle (MSP) entailment, based on a possibilistic approach. A compilation of default theories is proposed which provides polynomial algorithms for deriving MSP-conclusions.

Kern-Isberner and Ritterskamp also present advancement on default reasoning, but their approach is based on techniques from preference fusion. They consider the plausibility relation induced by each default rule on possible worlds, with the worlds that satisfy the rule preferred over those that falsify it. Fusing such simple preference relations gives rise to a complex preference structure on possible worlds that can be used for further conditional inferences. The resulting inference operation is shown to refine the well-known System Z, and to improve properties with respect to addition of irrelevant information.

When reasoning according to Dempster-Shafer theory, belief functions are usually combined using the unnormalized Dempster's rule. In their paper, Pichon and Denoeux present a new formal justification of the rule based on the canonical decomposition of belief functions, the least commitment principle, and the requirement of having the vacuous belief function as neutral element of the combination.

We acknowledge the following reviewers for their expertise, and for their careful, insightful and constructive reviews, which lead to the selection and publication of papers included in this special issue: Christoph Beierle, Salem Benferhat, Brahim Chaib-draa, Fabio Cozman, Andreas Herzig, Gabriele Kern-Isberner, Stbastien Konieczny, Philippe Leray, Liping Liu, Tsai-Ching Lu, David V. Pynadath, Paul Snow, Choh-Man Teng, and Pradeep Varakantham. 